\title{
Persistence for the Masses: RRB-Vectors in a Systems Language
}

\author{
JUAN PEDRO BOLÍVAR PUENTE
}

\begin{abstract}
Relaxed Radix Balanced Trees (RRB-Trees) is one of the latest members in a family of persistent tree based data-structures that combine wide branching factors with simple and relatively flat structures. Like the battletested immutable sequences of Clojure and Scala, they have effectively constant lookup and updates, good cache utilization, but also logarithmic concatenation and slicing. Our goal is to bring the benefits of functional data structures to the discipline of systems programming via generic yet efficient immutable vectors supporting transient batch updates. We describe a $\mathrm{C}++$ implementation that can be integrated in the runtime of higher level languages with a C core (Lisps like Guile or Racket, but also Python or Ruby), thus widening the access to these persistent data structures.

In this work we propose (1) an Embedding RRB-Tree (ERRB-Tree) data structure that efficiently stores arbitrary unboxed types, (2) a technique for implementing tree operations independent of optimizations for a more compact representation of the tree, (3) a policy-based design to support multiple memory management and reclamation mechanisms (including automatic garbage collection and reference counting), (4) a model of transience based on move-semantics and reference counting, and (5) a definition of transience for confluent meld operations. Combining these techniques a performance comparable to that of mutable arrays can be achieved in many situations, while using the data structure in a functional way.
\end{abstract}

CCS Concepts: • Software and its engineering $\rightarrow$ Data types and structures; Functional languages; • General and reference $\rightarrow$ Performance;

Additional Key Words and Phrases: Data Structures, Immutable, Confluently, Persistent, Vectors, Radix-Balanced, Transients, Memory Management, Garbage Collection, Reference Counting, Design Patterns, Policy-Based Design, Move Semantics, C++

\section{ACM Reference Format:}

Juan Pedro Bolívar Puente. 2017. Persistence for the Masses: RRB-Vectors in a Systems Language. Proc. ACM Program. Lang. 1, ICFP, Article 16 (September 2017), 28 pages.

https://doi.org/10.1145/3110260

\section{INTRODUCTION}

Immutability enables safe lock-free communication between concurrent programs. Persistence facilitates reasoning about change and is a fundamental to higher level reactive and interactive systems. A growing development community is increasingly interested in these properties, motivated by the horizontal scaling of our computing power, and the increased expectations that a wider and more diverse consumer base are putting on user interfaces. Many traditionally object-oriented programming languages are turning to a multi-paradigm approach embracing core functional programming concepts. It is the data structures that enable immutability and persistence at the scale of real world production systems.

This work is licensed under a Creative Commons Attribution 4.0 International License.

(c) 2017 Copyright held by the owner/author(s).

2475-1421/2017/9-ART16

https://doi.org/10.1145/3110260

Proc. ACM Program. Lang., Vol. 1, No. ICFP, Article 16. Publication date: September 2017. 


\subsection{Challenge}

Implementations of persistent data structures exist for various languages, both functional and otherwise. However, few attempts have been made to implement them in a language without a managed runtime or pervasive garbage collection. There are good motivations to try though. First, the systems programming community is adopting many techniques and principles from functional programming, as shown by Rust [Matsakis and Klock 2014] and the latest developments in recent $\mathrm{C}++$ standards. Second, a sufficiently general and efficient implementation could be integrated in the runtime of higher level languages (Lisps like Guile or Racket, but also Python or Ruby come to mind), allowing a wider audience to enjoy the benefits of these data structures. Doing so poses various challenges.

(1) Efficient persistent data structures require garbage collection. Without automatic garbage collection provided by the runtime, a reference counting reclamation scheme may be used. Doing so efficiently is challenging. Furthermore, when integrated in a runtime, it should be possible to leverage the garbage collector it provides, if one exists.

(2) Most immutable data structures are designed to store boxed values-i.e. pointers to objects allocated in the free store. ${ }^{1}$ Many performance critical applications require embedding the values in the data structure for further cache locality.

(3) An immutable interface may not always interact well with other components of a language that is not fundamentally functional. Furthermore, performance oriented systems developers might want to temporarily escape immutability when implementing transactional batches of updates to a data structure.

\subsection{Contributions}

We describe an implementation of RRB-Tree based vectors with transience in $\mathrm{C}++$. We overcome these challenges making the following contributions:

(1) The Embedding RRB-Tree (ERRB-Tree) data structure that efficiently stores arbitrary unboxed types $(\S 3)$.

(2) A tree traversal technique based on mutually recursive higher order position/visitors. It can be used to implement tree operations independently of optimizations that achieve a more compact representation of the tree (§ 4$)$.

(3) A policy-based design to support multiple memory management and reclamation mechanisms (including tracing garbage collection and reference counting) (§5).

(4) A model of transience based on reference counting (§ 6.2) and move-semantics (§ 6.3). These optimize updates by sometimes performing them in-place even when the containers are used in a functional style, making the performance profile is depend on the actual dynamic amount of persistence in the system.

(5) A definition of transience for all RRB-tree operations, including confluent meld operations ( $(6.4)$.

(6) An evaluation of the state of the art of Radix Balanced vectors, comparing it to various configurations of our implementation. This includes a discussion of the effects of reference counting, challenging the established assumptions on its suitability for the implementation of immutable data structures (§ 7).

Our implementation is libre software and freely available online. ${ }^{2}$

\footnotetext{
${ }^{1}$ Some implementations do embed basic numeric types, but since they have sizes similar to that of a pointer, they do not need to adapt the core algorithms.

${ }^{2}$ Immer: https://sinusoid.es/immer
} 


\subsection{Related Work}

1.3.1 Persistent Data Structures. Persistent data structures were originally studied as mutable data structures [Driscoll et al. 1986; Kaplan 2005]. Such data structures where the contents of two or more values can be merged in sub-linear time and space by sharing structure with all inputs are called confluently persistent [Collette et al. 2012; Fiat and Kaplan 2001]. Persistent data structures gained special momentum with Okasaki's [1999] seminal work on purely functional data structures. These are immutable and achieve efficient persistence via structural sharing. However, these have not reached mainstream programmers because of their small-node structures amortized via lazy evaluation, making them hard to implement efficiently in popular languages and modern architectures.

1.3.2 Cache Efficient Immutability. Phil Bagwell has done extensive work in building data structures with efficient cache utilization, including VLists [2002], Array Mapped Tries [2000] and Hash Tries [2001]. The latter two inspired the foundations of Clojure's [Hickey 2008] ubiquitous immutable vectors and maps. These quickly gained popular attention and various implementations are currently used in production in Scala, ${ }^{3}$ JavaScript, ${ }^{4}$ Python,,${ }^{5}$ and Java. ${ }^{6}$ [Steindorfer and Vinju 2015, 2016]

1.3.3 Relaxed Radix Balanced Trees (RRB-Trees). Bagwell and Rompf [2011] first proposed relaxing the Radix Balanced Tree structure in order to make the vectors from Clojure and Scala confluently persistent supporting $O(\log (n))$ concatenation. L'orange's [2014] masters thesis introduced transience for some operations, except concatenation and slicing, and it is one of the most thorough and intelligible descriptions of the inner workings of this data structure. Stucki et al. [2015] further developed the data structure adding amortized $O(1)$ updates via displays. RRB-Trees are often compared to other catenable sequences with efficient random access, such as Ropes [Boehm et al. 1995] $]^{7}$, Finger Trees [Hinze and Paterson 2006], and Chunked Sequences [Acar et al. 2014].

1.3.4 RRB-Trees in Systems Programming. L'orange [2014] implemented RRB-Trees in C, but they rely on Boehm and Weiser's [1988] conservative garbage collector and do not support unboxed types nor confluent transience. In parallel to this work, two other implementations of immutable vectors in $\mathrm{C}++$ have been published. ${ }^{8}$ These track garbage using atomic reference counts but do not support relaxed trees, transients, efficient embedding or customizable memory management.

\section{BACKGROUND}

\subsection{Radix Balanced Trees}

Radix Balanced Trees are inspired by Array Mapped Tries [Bagwell 2000] and were first introduced in the implementation of immutable vectors in Clojure [Hickey 2008]. They provide a good balance between structural sharing, random access complexity, and efficient cache utilization.

\footnotetext{
${ }^{3}$ Scala Standard Immutable Collection Library: http://www.scala-lang.org/docu/files/collections-api

${ }^{4}$ Immutable.js: https://github.com/facebook/immutable-js, Mori: https://github.com/swannodette/mori, Collectable: https: //github.com/frptools/collectable

${ }^{5}$ Pyrsistent: https://github.com/tobgu/pyrsistent

${ }^{6}$ Cyclops: https://github.com/aol/cyclops-react/

${ }^{7}$ Sean Parent claims in his talk "Inheritance Is The Base Class Of All Evil" that Ropes are used inside Photoshop to make their data model persistent. That talk has drawn the attention of a big part of the $\mathrm{C}++$ community towards immutability and value semantics, and is one source of inspiration for this work. https://channel9.msdn.com/Events/GoingNative/2013/ Inheritance-Is-The-Base-Class-of-Evil

${ }^{8}$ Steady: https://github.com/marcusz/steady, Immutable++ https://github.com/rsms/immutable-cpp
} 


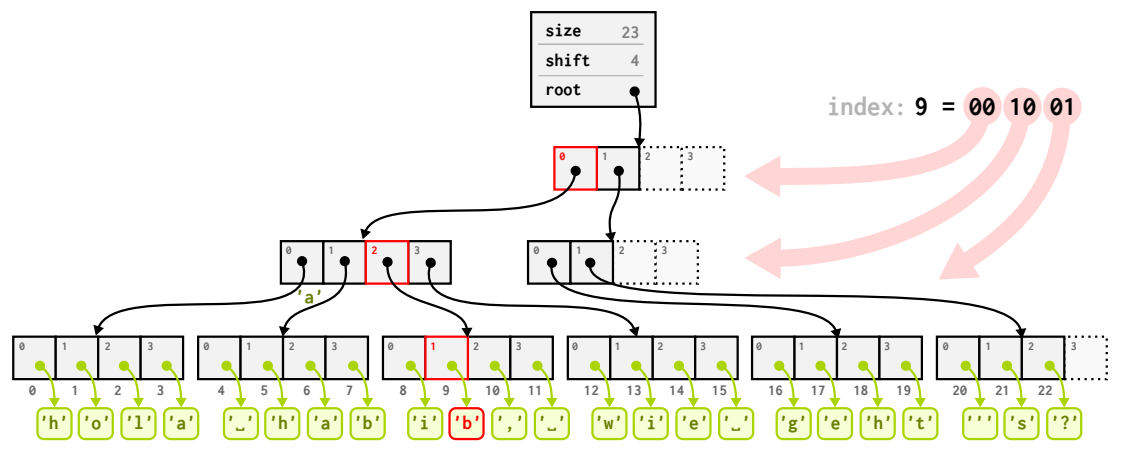

Fig. 1. A $B=2$ Radix Balanced Tree containing the 23 element string: "hola_habib, „wie_geht's?". The access path down to index 9 is shown in red.

As illustrated in figure 1, this is a tree structure where every inner node and leaf has $M=2^{B}$ slots (branches or elements respectively), where $B$ are the branching bits that characterize the tree. The rightmost path of the tree may contain nodes with less than $M$ slots. Every other node has exactly $M$ slots and is considered full.

2.1.1 Radix Search. We may locate the vector element at index $i$ in a radix balanced tree $t$ by traversing down the tree, where for every subtree $t^{\prime}$ with height $h\left(t^{\prime}\right)$, we descend to its child at offset $\left\lfloor i / M^{h\left(t^{\prime}\right)}\right\rfloor \bmod M($ radix search). Since $M$ is a power of two, we can simply divide the vector element index in $h(t)$ groups of $B$ bits and use each to navigate at each level of the tree. A common optimization is to keep track of the value $\operatorname{shift}(t)=B \times h(t)$ by storing it at the root. This denotes the depth of the tree while avoiding multiplications in tree traversals, using comparatively cheap bit-wise operations instead. Interestingly, an indexing mechanism very similar to radix search is commonly used to map virtual memory addresses to hardware addresses using multi level page tables [Drepper 2008]. ${ }^{9}$

A Radix Balanced Tree can also be considered a trie. If we think of numbers represented in base $M$ as strings with an alphabet of size $M$, an immutable vector of size $n$ is a trie containing every key in the range $[0, n)$ if we add left padding to the keys so they are evenly sized. This trie has a depth $h(T)=\log _{M}(n)$ and thus lookup runs in logarithmic time.

2.1.2 Branching Factor. We partially reproduced the results from [Bagwell and Rompf 2011; Hickey 2008; L'orange 2014; Stucki et al. 2015] claiming that $M=32(B=5)$ is a sensible choice on modern architectures. ${ }^{10}$ With such high branching factor elements are stored in contiguous blocks spanning a few cache lines and thus the CPU caches are used effectively and the structure can be iterated fast. Furthermore, such tree containing $2^{32}$ has only depth of 7 , this is, it contains every element that is addressable using 32 bit integers yet search elements in only 7 steps. When dealing with lots of data, other factors (like working set size vs cache size) have an impact orders of magnitude larger than the depth of the tree. For this reason, in practice, it is useful to think

\footnotetext{
${ }^{9}$ In fact, the way Linux implements virtual memory via copy-on-write can be thought of as massive persistent vector, where memory pages are leaf nodes, and page tables are inner nodes, and each fork ( ) creates a new version of the data structure. So we have the operating system managing memory as massive persistent vectors at one end, and at the other end language users build their programs out of small persistent vectors and hash-tables. We could fill the sandwich adding a persistent vector based memory allocator/garbage collector, fulfilling the wild fantasies of the authors-maybe a future work proposition?

${ }^{10}$ Informal experiments using our implementation show that the values 4, 5 or 6 all sensible, having different impact on different operations. The optimal choice depends on the particular hardware, workload, and memory management strategy.
} 


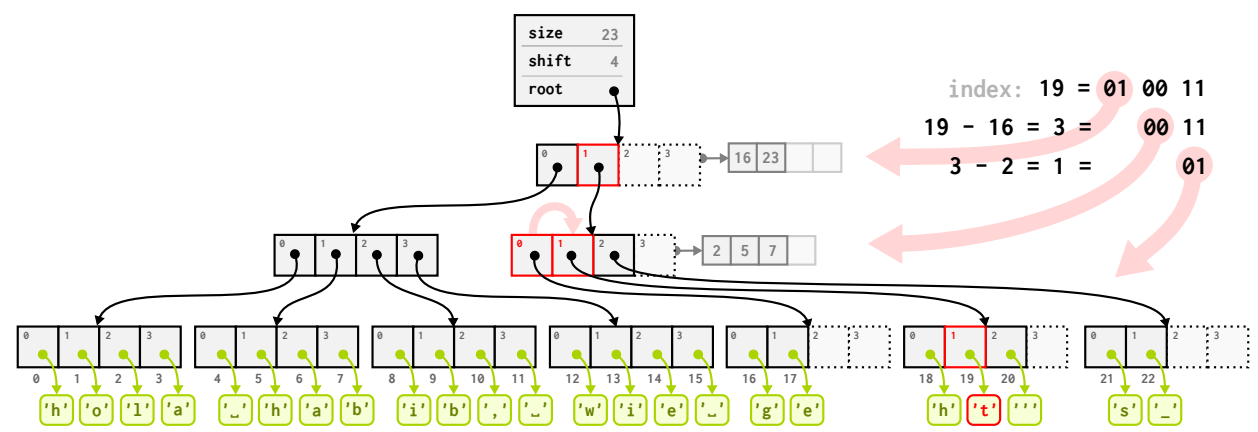

Fig. 2. A $B=2$ RRB-Tree containing the 23 element string: "hola_habib, „wie_geht's?". The access path down to index 19 is shown in red. At level 2, an extra step is taken in order to find the right subtree.

of radix search complexity as constant. As such, it is often advertised that Radix Balanced based vectors support effectively constant random access.

\subsection{Relaxed Radix Balanced Trees}

Regular Radix Balanced Trees provide effectively constant random access, updates, appends, and right slicing (take). However concatenation is proportional to the size of the right argument, and left slicing (drop) is proportional to the number of remaining elements. This is caused by the strict balancing required for radix search. By relaxing this requirement, Relaxed Radix Balanced Trees (RRB-Trees) support $O(\log (n))$ concatenation, left-slicing, and insertion at random locations [Bagwell and Rompf 2011; L'orange 2014; Stucki et al. 2015].

Regular Radix Balanced Trees are a strict subset of valid RRB-Trees. RRB-Trees introduce a new kind of node, relaxed inner nodes, whose children are allowed to contain less elements than those required by radix search. These nodes have an array with the accumulated sizes of its subtrees as illustrated in figure 2.

Relaxed Radix Search sometimes needs to step through the size array to look up the actual subtree containing the searched element. When it descends to a regular subtree, it falls back to regular radix search-regular subtrees can not contain relaxed subtrees. A rebalancing concatenation operation can be defined in $O(\log (n))$ that bounds the amount of extra search steps required such that random access remains effectively constant in the resulting structure (albeit with a higher constant).

This data structure provides a very good compromise for big vectors in which insertions at arbitrary positions are frequent. It also opens new opportunities for concurrent and parallel programming. Operations that change the size of the vector, such as filtering, can be parallelized by processing the vector in chunks and then concatenating the results [Prokopec 2014; Stucki et al. 2015].

\subsection{Optimizations}

2.3.1 Transience. By default, persistence is achieved in RRB-Trees via immutability. Because data is never modified in-place, updates involve copying the whole path down to the updated element. While this is desired most of the time, it is overkill when a function produces many intermediate versions that are immediately discarded.

Clojure proposes a pair of polymorphic functions (transient $v$ ) and (persistent! $t$ ). The first one returns a mutable (i.e. transient) version of the immutable collection $\mathrm{v}$, for which mutating operations do exist. The second returns an immutable snapshot of the transient collection $t$ 


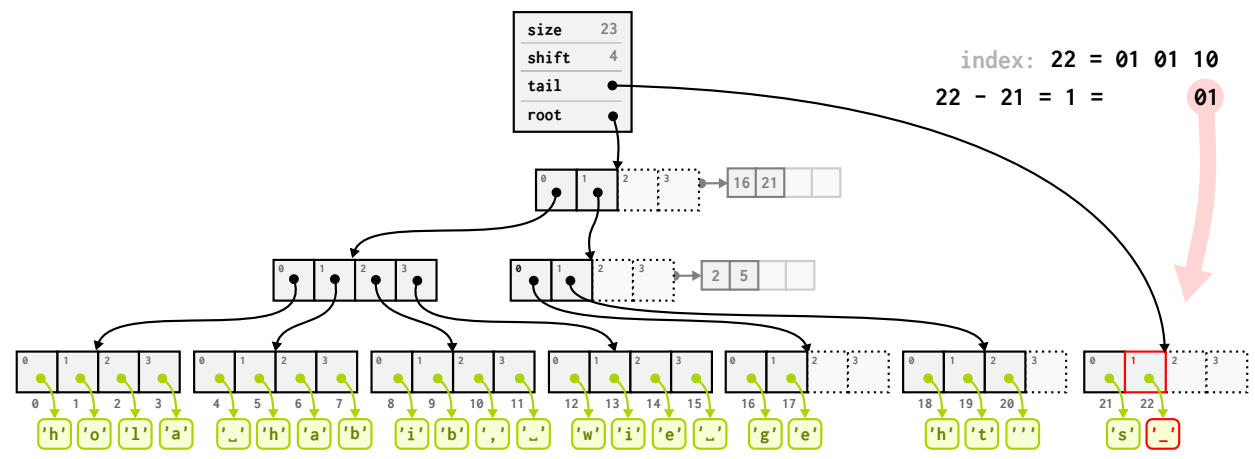

Fig. 3. A $B=2$ RRB-Tree with off-tree tail containing the 23 element string: "hola_habib, „wie_geht's?". The access path down to index 22 is shown in red. Note that since the element lies in the tail, the tree does not need to be traversed.

and invalidates t. Both operations are $O(1)$. Updates on a transient collection are done in-place whenever possible, doing copy-on-write to ensure the immutability of the adopted contents. We further discuss transience in $\S 6$.

2.3.2 Off-tree Tail. When using immutable vectors, users may expect fast appends, as it is the case for mutable vectors. The most common optimization when implementing Radix Balanced Trees is to keep the rightmost leaf off-tree and point to it directly from the root, as shown in figure 3. In most cases only the tail needs to be updated to append a new element. Once every $M$ appends the tail gets full-it is inserted into the tree and a new tail is created. Appends become significantly faster this way and our implementation includes this optimization.

2.3.3 Left Shadow. We saw that the rightmost path may contain nodes with less than $M$ slots. A way to think about this is to consider that every Radix Balanced Tree $t$ does virtually contain $M^{h(t)}$ elements. When the size of the vector is not a power of $M$, the rightmost $M^{h(t)}$ - size elements are null. The representation is compressed by not storing all these empty rightmost branches in memory. This is the right shadow of the tree.

Instead of projecting our vector elements in the indices [0, size) of the tree, we could as well use some other [ f irst, last) range, thus creating another shadow on the left. In this way, it is possible to implement effectively $O(1)$ drop and prepends with regular Radix Balanced Trees. This optimization is implemented in Scala [Stucki et al. 2015]. We chose not to implement this optimization, but we are considering it for future work.

2.3.4 Display. The display is a generalization of the off-tree tail mechanism that leverages the spatiotemporal locality of updates. The core idea is to establish a vector element as the focus. The whole path down to this index (the display) is kept off-tree and stored directly at the root. It was found that by using an exclusive-or (xor) operation between two indexes it is easy to find their common ancestor in the tree. Updates close to the focus become faster because only the sub-path after the common ancestor needs to be updated. Vector manipulations change the focus to the index where the update occurs. In this way, sequential updates become amortized $O(1)$. We experimented with this optimization for a while, but decided not to include it in our final implementation, because:

(1) The root node becomes bigger because it stores a whole path. This means that if we store it on the stack or unboxed in some container, it becomes more expensive to copy it: both 
because of the bigger size, but also because the reference counts of all nodes in the display need to be touched.

(2) Implementation complexity is increased. Some assumptions are invalidated (e.g. now some references inside the tree are null when they belong to the display) adding further conditional checks and overhead to operations that do not benefit from the display.

(3) Transients are an alternative way of improving the peformance of sequential updates. While less general and pure, they provide better performance when applicable (§ 7.2.4).

\section{EMBEDDING UNBOXED VALUES}

\subsection{Problems with Boxed Values}

All evaluated RRB-Tree literature and implementations assume boxed values. ${ }^{11}$ This is, the container does not manage the memory where the actual element values are stored. Instead, the values are located in separate objects (i.e. boxes) in the free store. The leaf nodes of the RRB-Tree just store pointers (i.e. references) to these objects, not the object themselves. This degrades performance in many situations:

(1) The elements are stored in separate objects that are potentially distant in memory. The extra indirection and the lack of locality result in suboptimal usage of the CPU caches, causing slower accesses.

(2) Every free store object requires extra memory usage, because of the extra pointer, but also because of the cost of boxing itself. ${ }^{12}$

\subsection{Naive Unboxing}

In system languages supporting manual memory management, containers often embed the contained values directly in the data structure, thus tackling the problems above.

A naive way of embedding values in RRB-Trees would be to store the contained objects directly at the leaves. In $\mathrm{C}++$ we could represent these leaves as standard arrays-i.e. T[M] or std: : array $<\mathrm{T}, \mathrm{M}>$ for an element type T. However, this approach has one fundamental flaw: the size of the leaf nodes is now proportional to the size of $\mathrm{T}$ and normally different from the size of the inner nodes.

The established result that $B=5$ provides efficient updates does no longer hold. It does not suffice to compute an alternative better $B$ for each different sizeof $(T)$. Note that for user defined types, the size of $T$ is unbounded-they may write types as big as they want. For bigger types, we may try to compensate lowering the branching factor. But doing so we also increase the depth of the tree, damaging random access performance. For very big types, we end up with the degenerate case $B=1$ (a binary tree) where the benefits of the RRB search are completely lost.

\subsection{Embedding Radix Balanced Trees}

We propose a variation called Embedding (Relaxed) Radix Balanced Trees or ERRB-Trees.

3.3.1 Definition. An ERRB-Tree is characterized by two constants, the branching bits $B$ and the leaf branching bits $B_{l}$, that relate to the branching factors $M=2^{B}$ and $M_{l}=2^{B_{l}}$ respectively. Its structure is similar to that of a standard RRB-Tree, but while inner nodes contain up to $M$ slots, leaf nodes now contain up to $M_{l}$ slots. Figure 4 shows an example of such tree and how addressing works in this case.

\footnotetext{
${ }^{11}$ Some implementations, like Clojure, have partial support for unboxed primitive types. However, in most architectures the size of primitive types is similar to the size of pointers, thus not rising the problems discussed here.

${ }^{12}$ Depending on the implementation, every free store object might have associated free list pointers, object size, GC mark/lock flags, padding due to bucketing or fragmentation, etc.
} 


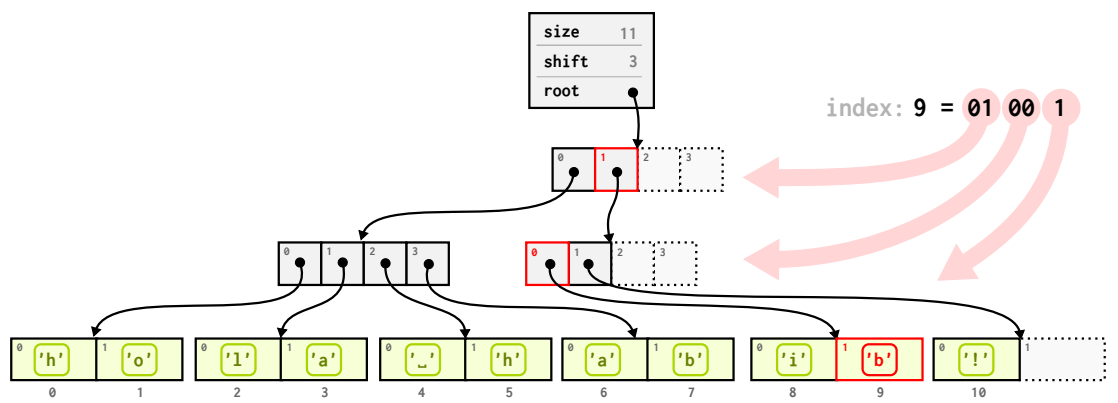

Fig. 4. A $B=2, B_{l}=1$ ERB-Tree containing the 11 element string: "hola_habib!". The access path down to index 9 is shown in red. Every element is twice the size of a pointer (we could imagine we are storing a UTF-32 string in a 16-bit architecture) and the contained objects are embedded directly in the leaves.

Under the new structure, looking for vector element at index $i$, the offset in the leaf array is $\left\lfloor i / M_{l}\right\rfloor$. Thus, the shift $(t)$ is now defined as:

$$
\operatorname{shift}(t)= \begin{cases}B_{l}+B \times(h(t)-1) & \text { if } h(t)>0 \\ 0 & \text { if } h(t)=0\end{cases}
$$

Special care has to be taken to accomodate the base case of recursive tree traversals to this new shift definition. Most algorithms are otherwise very similar to those of the original RRB-Trees. Listings $3.1 \mathrm{a}$ and $3.1 \mathrm{~b}$ compare potential $\mathrm{C}++$ definitions of naive and embedding RRB-Trees and their respective random access operations.

3.3.2 Choosing $B_{l}$. The question remains: what are the best values for $B$ and $B_{l}$ ? Intuitively, we expect the answer to depend on sizeof $f_{T}$. Experimentally we can show that $B=5$ remains valid, but $B_{l}$ should be chosen such that leaf nodes are similar in size to inner nodes. The size of inner nodes depends only on $B$, since the size of a data pointer $\left(\right.$ sizeof $\left._{*}\right)$ is usually fixed for a given CPU architecture. For a given $B$ and $T$, we may derive the branching bits at the leaves as:

$$
B_{l}^{\prime}(B, T)=\left\lfloor\log _{2}\left(\frac{\text { sizeof }_{*} \times 2^{B}}{\text { sizeof }_{T}}\right)\right\rfloor
$$

Note that the choice to floor the result might seem rather arbitrary. This ensures that sizeof $f_{T} \times$ $2^{B_{l}} \leq$ sizeof $_{*} \times 2^{B}$, this is, that leaf nodes are at most as big as inner nodes. This property enables reusing buffers used to store all kinds of vector nodes across different value types (§ 5.3).

Also, we see that $B_{l}^{\prime}=0$ when sizeof $f_{T}>$ sizeof $_{*} \times 2^{B-1}$. In this case, leaf nodes contain only one element and no bits are used to address into it. Such ERRB-Tree is equivalent to a RRB-Tree with the same $B$ (off-tail optimizations not considered).

In our $\mathrm{C}++$ implementation, both $B$ and $B_{l}$ can be customized by passing template arguments. This allows the user to optimize the data structure for architectures not considered by the authors, or to reproduce the results here described. Otherwise, they default to $B=5$ and $B_{l}=B_{l}^{\prime}(B, T)$. The latter is derived at compile time when the containers is instantiated for a given element type $T$.

\section{MINIMIZING METADATA}

\subsection{Incidental Metadata}

A naive implementation of RRB-Trees stores the following two pieces of metadata in every node: 


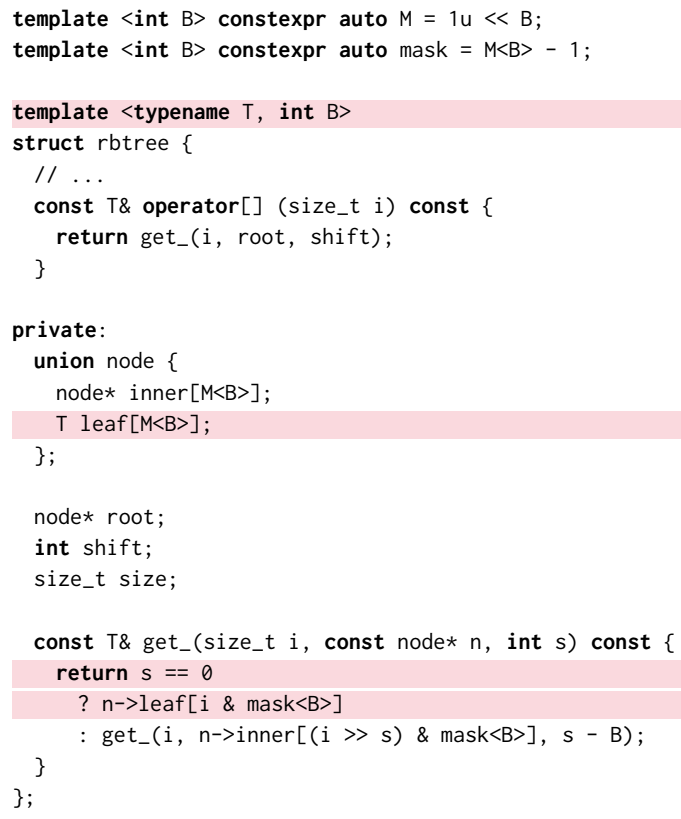

(a) Naive Radix Balanced Tree

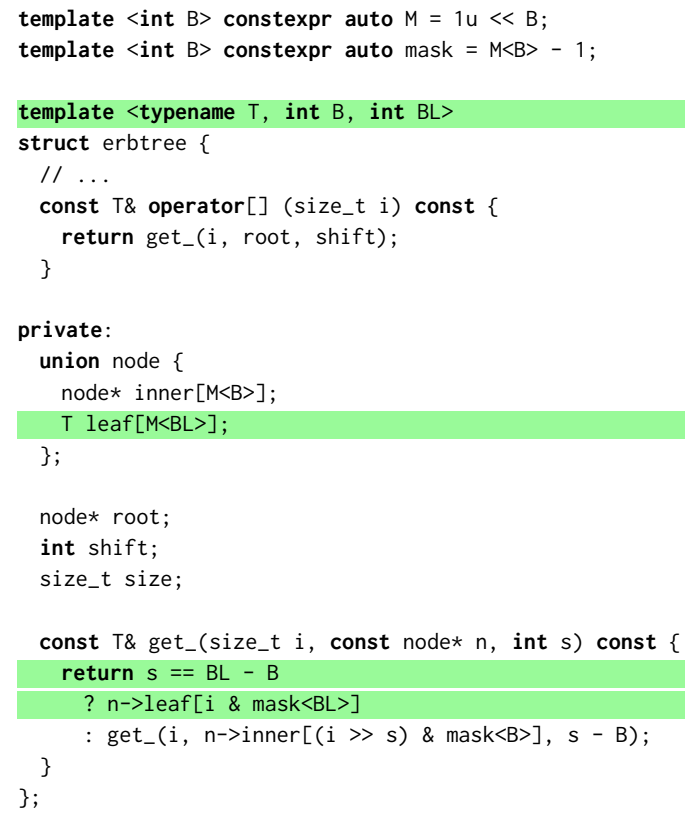

(b) Embedding Radix Balanced Tree

Listing 3.1. A potential definition Naive and Embedding RRB-Trees

(1) The type of node. This is, whether it is a leaf or inner node (regular or relaxed). This may happen implicitly-i.e. it is added by the compiler when using runtime polymorphism, in the form of a pointer to a v-table, type tag, or alike.

(2) The number of slots in the node. In many languages this is added implicitly too-for example, Java arrays provide an length member. ${ }^{13}$

\subsection{Fundamental Metadata}

For relaxed inner nodes, some kind of metadata is unavoidable. This is so because (1) they may have a variable number of sub-nodes, and (2) they have to be distinguished from regular inner nodes. An inner node thus has a member that may point to an object containing the number of children and the cumulative size of each subtree. This pointer is null in regular inner nodes. In most implementations, this pointer is stored at the end of the children array. We store it before the children array-experimentally we saw that it makes no significant performance difference ${ }^{14}$ but it makes the position fixed and independent of the number of elements.

\subsection{Removing Incidental Metadata}

4.3.1 Type Information. No other information about the type of node is fundamentally required, because we can distinguish inner from leaf nodes by their distance to the root relative to the shift. But as long as no type metadata exists, nodes do not know how to destroy themselves. This means that

\footnotetext{
${ }^{13}$ Whether this adds an overhead depends on the implementation. Very often, the free store needs to know the size of the node anyways, but this information is lost in abstraction in languages like $\mathrm{C}$ or $\mathrm{C}++$.

${ }^{14} \mathrm{Has}$ a tiny advantage for some operations, a tiny disadvantage for others.
} 
While efficient, this code suffers from significant duplication. The do_update and do_update_full functions are identical excepting for (1) the computation for the slot count, and (2) the the recursive call down to the next child. The problem gets worse when we introduce relaxed nodes in RRB-Trees and binary operations like concatenation. To solve this problem we introduce the notion of positions.

\subsection{Position Based Traversals}

A position is an object that contains a reference to a node alongside the metadata needed to do useful things with it. It is not stored in the tree, but created on demand during traversals. A position supports a series of higher order operations to visit its children. These operations use the context they have to instantiate the most efficient position type for the child, and pass this instance to a visiting operation that it took as argument. Via mutual recursion between positions and visitors, any kind of traversal can be described.

A visitor may be defined as one generic function that is applied to any kind of node. Recursive traversals terminate because higher order operations for leaf nodes do nothing. However, most useful visitors are implemented as two or three functions that distinguish leaf or inner nodes (regular or relaxed).

Listing 4.2 shows how the update() operation may be implemented in terms of the position framework. Appendix A shows a possible implementation of a position framework supporting this kind of operation. Note that this vistor is defined for Embedding RRB-Trees, but only positions and the node copying operations are concerned with the details of embedding and relaxation.

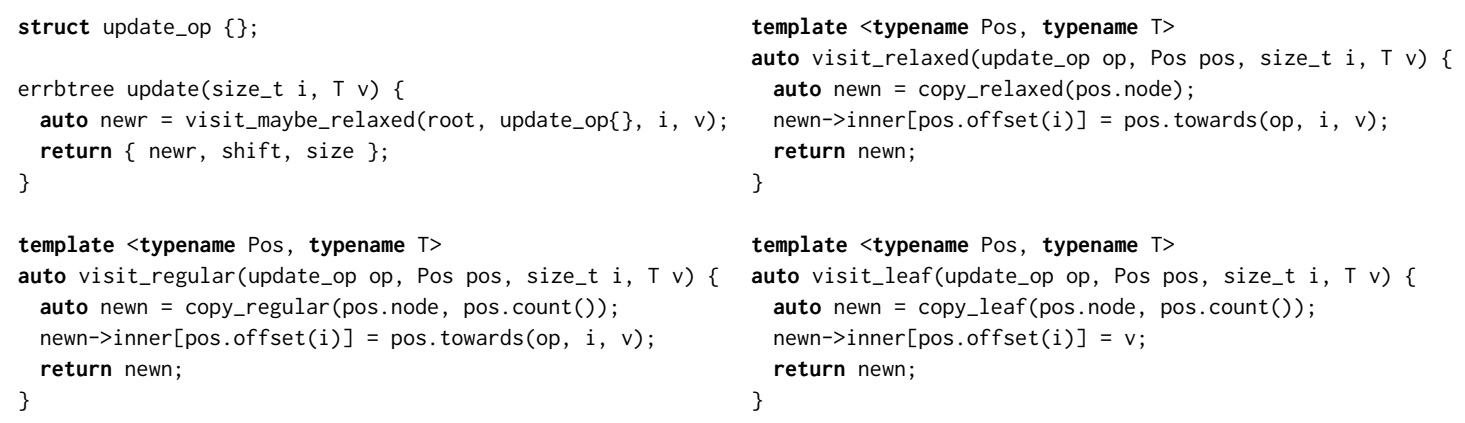

Listing 4.2. The update() (E)RRB-Tree operation using positions.

All the bit wizardry is hidden in the positions, and the traversal can be optimized without changing the visitor. The operation is not concerned anymore with the details of how to navigate through the tree. Instead, it focuses on what it needs to do to each node in order to produce a new structure. No combinatorial explosion happens between the types of nodes and the types of positions. When implemented in this way, changing an RRB-Tree into an ERRB-Tree structure is simple.

This code is also as performant as the hand-written traversal without positions. Note that all dispatching is done statically. The recursive visitor takes the positions as a template argument, and it is instantiated at compile time for all possible positions in the traversal. When instantiating the visitor in listing 4.2 against the position framework in appendix A, a call graph as shown in figure 5 is produced. All definitions are visible to the compiler allowing inlining and other optimizations. 


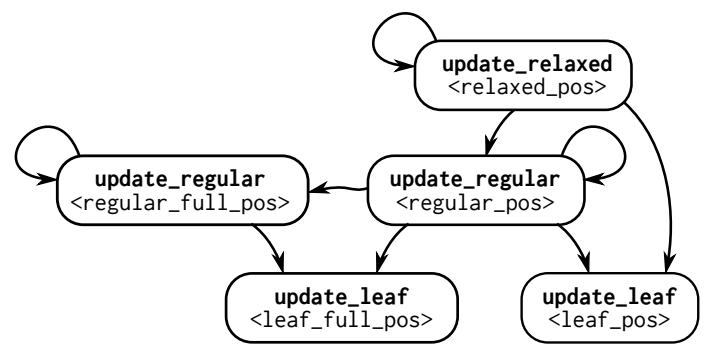

Fig. 5. Instantiated call graph for the update() operation using positions over an (E)RRB-Tree.

\section{MEMORY MANAGEMENT}

A garbage collection mechanism is required for immutable persistent data structures to be effective. As shown, when an operation updates the data structure, new nodes are allocated to copy the changed parts of the data structure, but the new value also references parts that did not change. Eventually, when old values are not used anymore, a node may loose any references to it and its memory should be recycled.

\subsection{Garbage Collection in $\mathrm{C}++$}

$\mathrm{C}++$ does not provide any pervasive garbage collection mechanism by default. These three mechanisms are often used instead:

(1) Reference counting. This is the most common technique. It can be implemented using $\mathrm{C}++$ constructor/destructors and its determinism plays well with the rest of the language. The standard library provides an opt-in generic reference counting pointer type (shared_ptr).

(2) Uncooperative garbage collection. A common implementation is Boehm's conservative garbage collector [Boehm and Weiser 1988]. Composing GC-based objects into objects that use malloc is problematic because the GC can not see the malloc-ated memory. ${ }^{16}$ Specially troublesome is the fact that there is no way to efficiently and deterministically call destructors of $\mathrm{C}++$ objects. Still, since $\mathrm{C}++11$ the standard allows such garbage collectors via an optional API [Boehm et al. 2008].

(3) Cooperative garbage collection. This is a common solution when writing $\mathrm{C}$ extensions to higher level languages. For example, GNU Guile provides an API for custom C types to cooperate in the mark phase of the GC [Galassi et al. 2002]. Racket [Flatt and PLT 2010] supports a compacting GC but defines an $\mathrm{API}^{17}$ with sufficient semantics for $\mathrm{C}$ extensions to cooperate [Rafkind et al. 2009]. Python ${ }^{18}$ or Ruby [Grimmer et al. 2015] are two other examples with similar approaches.

\subsection{Policy Based Design}

We use policy based design [Alexandrescu 2001] to support all these mechanisms without implementing the data structure multiple times. A policy is a type modelling a concept that factors some aspects of the behavior of some other type. A type takes in policies as template arguments that are bound and monomorphized at compile time, allowing the compiler to inline calls or remove empty

\footnotetext{
${ }^{16}$ Workarounds exist for this problem. With libgc one can use GC_malloc_uncollectable instead of std: : malloc where memory is managed manually.

${ }^{17}$ http://docs.racket-lang.org/inside/im_memoryalloc.html

${ }^{18}$ https://docs.python.org/3/extending/
} 
objects $^{19}$. It is sometimes thought of as a compile-time version of the strategy pattern [Gamma et al. 1995]. A good factoring into policies enables extensibility without a performance penalty and it is thus useful to enable the user make their own trade-offs when configuring performance sensitive aspects of some type. Listing 5.1 shows two policies for reference counting as implemented in our system.
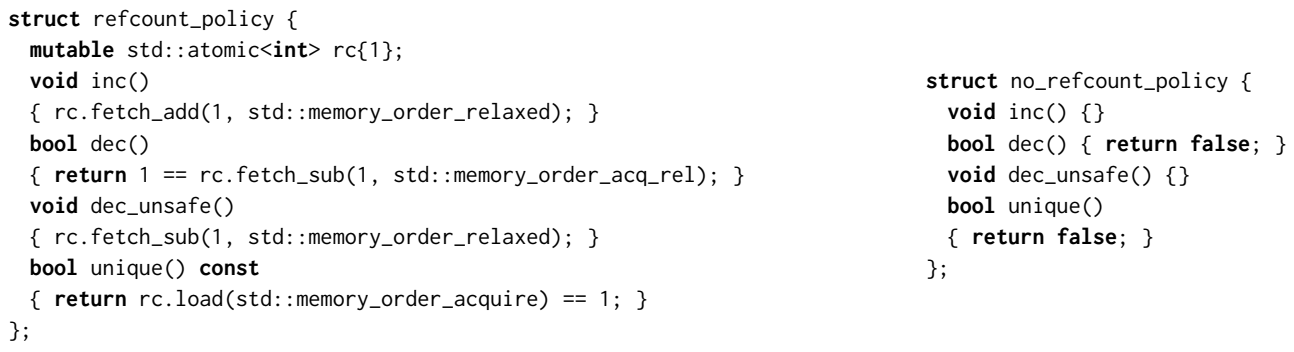

Listing 5.1. Two reference counting policies. The refcount_policy enables thread-safe reference counting via an atomic integer count. The no_reference_counting is a no-op policy to be used when some other garbage collection is available. Our system provides an additional unsafe_refcount_policy for singlethreaded systems.

\subsection{Memory Policies}

In our system, users can customize various aspects of the memory layout and management by passing a memory_policy<...> as one of the template arguments of the container. A memory policy takes the following arguments:

(1) A HeapPolicy specifies how memory should be allocated and released. It allows, for example, to exchange standard malloc with libgc [Boehm and Weiser 1988].

Furthermore, our heap policies use memory allocator layering [Berger et al. 2001] to be able to define memory allocators out of composable building blocks. We provide heaps adaptors to enable both global and thread-local free-lists.

(2) A RefcountPolicy like the ones in listing 5.1.

(3) A TransiencePolicy used to implement transience when reference counting is not available (§ 6.1.1).

(4) A prefer_fewer_bigger_objects flag determining the layout of inner nodes as shown in figure 6.

(5) A use_transient_rvalues flag determining whether $r$-values should be considered transients (§ 6.3).

Note that not all parameters are completely independent-this is, there are some combinations of policies that do not make sense. For example, using a libgc_heap only a no_refcount_policy and a gc_transience_policy should be used. Likewise, no_transience_policy should be used whenever a reference counting mechanism is enabled. Some of these dependencies are captured by meta-functions that default-initialize the unspecified arguments of the memory policy.

${ }^{19}$ When combine with techniques like empty based optimization 


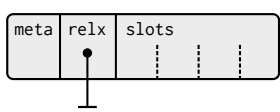

(a) Inner node

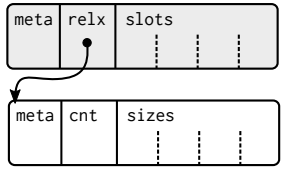

(b) Relaxed node

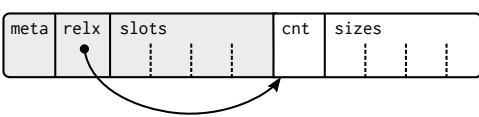

(c) Relaxed node when

PreferFewerBiggerObjects=true

Fig. 6. Inner node layouts. Regular inner nodes do not store the size of the subtrees, thus the relx pointer is null. A relaxed node may or may not have the size array allocated in the same memory object. In the former case, the size array needs its own meta to track reference counts or transient ownership. In the latter case, the size array can not be shared between nodes, making the update() slower, but improving traversals and reducing allocations in other update operations.

\section{TRANSIENCE}

\subsection{Background}

While RRB-Trees perform very well for a persistent data structure, they are suboptimal when persistence is not required. This happens when writing pure functions that perform multiple updates to a data structure but only return the last version. From the point of the view of the caller, the function is a transaction and we can only observe the accumulated effects of the whole operation. We may persist its inputs and outputs, but intermediate results produced inside the function are necessarily forgotten when it returns. An example of such suboptimal operation is shown in listing 6.1a, which defines a $\operatorname{iota}(v, f, l)$ that appends all integers in the range $[f, l)$ to the immutable vector $v$.

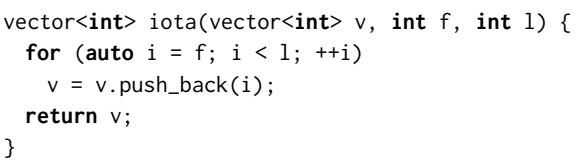

(a) Suboptimal iota()

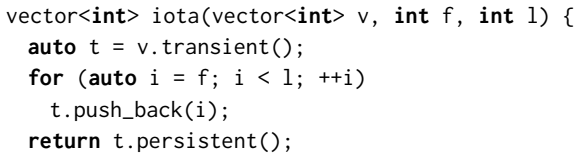

(b) Efficient iota() using transients

Listing 6.1. Populating an immutable vector

Clojure solves this problem by introducing the notion of transients. A transient container can be constructed in $O(1)$ from its persistent counterpart by merely copying a reference to its internal data. However, the transient has different semantics, such that update operations invalidate the container supplied as argument. This allows transient operations to sometimes update the datastructure in-place, stealing the passed in tree and mutating its node objects. Still, the transient has to preserve the immutability of the original persistent value whose contents it adopted at the beginning of the transaction.

6.1.1 Copy on Write. To be able to track which nodes it can mutate in place, the transient is associated with a globally unique identifier that is generated at the beginning of the transaction. We then proceed using a copy-on-write strategy. In a transient update operation, before mutating a node in place, we check whether the node is tagged with the current transient identifier-i.e. we check if the transient owns the node. Only if it owns the node is it allowed to modify the node in-place-otherwise a new copy is made, tagged with the transient identifier. Update operations 
do this for every node in the path down to the affected leaves, thus making sure the mutations have no visible effects outside of the transient. A transient can be converted back into a persistent value, invalidating the transient. Thanks to this invalidation, the operation can be performed in $O(1)$ without cleaning up the tags-a new transient is going to have a different identifier, so it is impossible for newer transients to mutate those nodes that are remain tagged with the identifier of the finished transient. In our system, the transience policies (see appendix B for an implementation) describe how these identifiers are created and compared.

6.1.2 Interface. In Clojure, transient operations are not referentially transparent, even though functions that only use them internally can be so. ${ }^{20}$ However, transients can be modelled in a pure language supporting affine types [L'orange 2014; Walker 2005].

In $\mathrm{C}++$ it feels natural to encapsulate transients behind an explicitly mutable interface as exemplified in listing $6.1 \mathrm{~b}$. This makes the invalidation of the previous state more obvious, hopefully lowering chances for programming mistakes. Also this interface is compatible with generic standard library algorithms and components which expect mutable containers. In this way, persistent vectors become a first class citizen of the language. In section $\S 6.3$ we present an alternative interface that does not sacrifice a functional style.

\subsection{Reference Counting}

The ownership tracking mechanism described above is redundant when combined with reference counting. Instead of generating transient identifiers, we can make a node $t$ eligible for in-place mutation when its reference count is 1 and every node in the path up to the root also has a reference count of 1 . This means that the path is only referenced by the transient and the effects of modifying it in-place are not visible outside of the transient.

The two ownership tracking rules can be combined into one. Algorithm 6.1 shows the general structure of a copy-on-write update operation that can operate under either mechanism. Each of the rules are implemented via policies, and they can be disabled by passing in a no-op policy that the compiler will inline away. Normally, only one of these two policies should be enabled for a given instance-using both mechanisms at the same time is harmless albeit inefficient and redundant.

\subsection{Move Semantics}

6.3.1 R-value References. C++ implements the notion of move semantics via $r$-value references [Hinnant et al. 2004]. Typically a variable can be bound to a either (1) a value (type of the form T), this is, an new object whose lifetime is determined by its lexical scope, or (2) a reference (type of the form T\&), this is, an alias to some other existing object. In $\mathrm{C}++11$ (3) $r$-value references were introduced (type of the form T\&\&), this is, a special reference that can only alias anonymous objects-temporaries, like the object containing the result of an expression.

$\mathrm{R}$-value references are useful when combined with overloading. For some operations, it is common to provide two overloads, one that takes in an r-value reference of some type (T\&\&), and another one that takes in read-only references of that type (const T\&). In general the latter overload will be used, but when some anonymous object is passed as argument the former takes precedence. The T\&\& overload can often be implemented more efficiently because, since the reference is known to be bound to an anonymous object-this is, to an object that that does not have any other alias and thus is invisible outside of this scope. This allows to steal parts of the internal representation of the argument in the process of producing its own results. This is, while in a const T\& overload

\footnotetext{
${ }^{20}$ In Clojure, there are runtime checks protect against using using an invalidated transient or sharing a transient across multiple threads.
} 

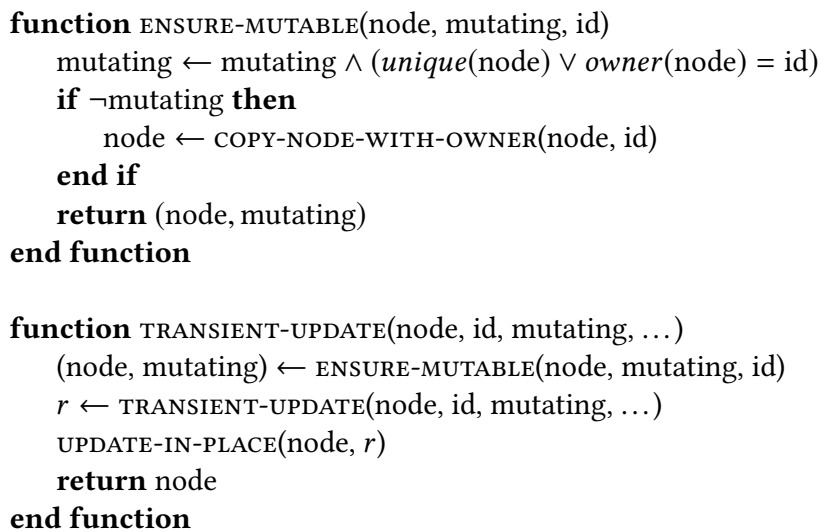

Algorithm 6.1. Structure of a transient operation. The unique(node) expression tests whether a node's reference count is 1 , and owner (node) returns the identifier associated to the transient owning the node.

the passed in objects need to be deeply copied when deriving new results from the passed in values, the T\&\& moves the passed in contents into the results, hence the name move-semantics for this general approach.

A truly anonymous $r$-value could in theory only be used once, thus forming a special kind of affine type. Affine types where used in the Rust programming language to model fully type safe move-semantics that unlike $\mathrm{C}++$ are not based in $\mathrm{r}$-value references [Matsakis and Klock 2014].

In $\mathrm{C}++\mathrm{r}$-values references are not truly unique aliases because (1) the destructor is unconditionally called at the end of its lifetime, even after it was moved, and (2) the programmer can also cast a named variable (an l-value) to an r-value using the std: : move() function. Even though an object is left in an unspecified state after being moved from, it is valid in the sense that the destructor must still succeed. The compiler will not check that a named object that has been moved from is not used again. Furthermore, it is common to define the assignment operator such that moved-from objects can be reassigned to give them a specified and known state.

6.3.2 R-value Transients. These semantics allow us to say that an $\mathrm{r}$-value of a persistent container is a transient $r$-value. For every operation in the container, an overload for $r$-value references is provided that optimizes updates using the transient rules. Listing 6.2 show two examples where transient $r$-values are manipulated.

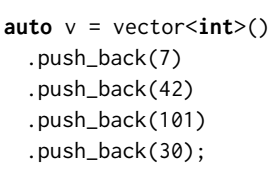

(a) Chaining r-value temporaries

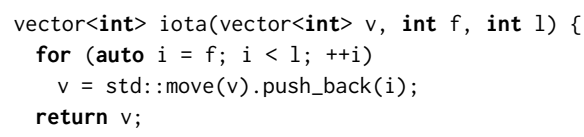

(b) Casting I-values to r-values

Listing 6.2. Manipulating $r$-value transients. On the left, every push_back call is applied to temporaries and thus the transient rules are used to perform in-place updates without any intervention from the programmer. On the right, by explicitly moving $v$ into the push_back call, this implementation of iota has the same performance as that in listing $6.1 \mathrm{~b}$.

The applicability of this technique is not exclusive to $\mathrm{C}++$. Notably, Rust is a systems programming language that includes type safe move semantics (borrowing) modelled after affine types, but 
its lack of overloading means that the programmer would always need to explicitly note which version (transient or persistent) of an operation to pick-even though the compiler would most often protect them from picking the wrong one. We dream of a language that provides the transparency of overloaded $\mathrm{r}$-value references (like $\mathrm{C}++$ ), while removing the burden of explicitly moving variables when they are last used by using stronger and more type safe scoping rules (like Rust).

6.3.3 Ownership Tracking. Since l-values can be converted into r-values, we still need to track node ownership to ensure we do not modify aliased nodes. When using identifier based ownership tracking, we would need to generate a new identifier whenever we alias a persistent vector (in $\mathrm{C}++$ terms, when copy it). This means that in-place modifications would happen only from the second operation that is applied consecutively on a $\mathrm{r}$-value transient chain. Only programs with lots of batch updates would benefit from this-for most programs, we speculate that the cost of generating new identifiers all the time out-weights the potential gains.

However, reference counting keeps an accurate count on the aliasing of a node all the time anyways. No extra runtime cost is required to optimize updates on r-values, with potentially very high gains. Our implementation uses r-value transience whenever reference counting is enabled. This can be controlled by the user via the use_transient_rvalues switch of the memory_policy $(\S 5.3)$.

6.3.4 Costs. To enable in-place push back, the rightmost path of the tree needs to keep enough space allocated in the nodes to write the new data. In the worst case, this has a $M \times \log _{M}(n)$ memory overhead. While this is negligible for non-trivial vectors, it might have an impact on an application that uses lots of small vectors where $n \ll M$. A solution could be to use an exponential growth mechanism for rightmost nodes, similar to that used for non persistent vectors. Sometimes the programmer may as well juse use immutable arrays when $n<M$.

\subsection{RRB-Tree Transience}

6.4.1 Background. Transients were first introduced in Clojure for regular Radix Balanced Tree based vectors. Later, when they implemented RRB-Trees ${ }^{21}$ they only added support for transient push, take, and update. They do not store the owner id in the size array of relaxed nodes. Thus, the size array needs to be copied unconditionally when updating an aliased node, even when the sizes of the sub-trees do not actually change.

L'orange [2014] proposes storing the owner id in the size array, which increases the level of structural sharing after an update operation. Furthermore, when possible, they convert an editable regular inner node into a relaxed node by assigning a size array in-place. However, their implementation only supports transient push and update.

We implemented transient versions of all RRB-Tree operations, using either of the previous approaches depending on whether the size array is embedded or not (see figure 6). For most operations, a transient alternative can be designed by systematically applying the structure described in algorithm 6.1. However, concatenation requires special treatment.

6.4.2 Confluent Transients. Borrowing the terminology from Fiat and Kaplan [2001], concatenation is a meld operation-i.e. an operation that combines at least two persistent values into one. RRB-Trees is confluently persistent because it supports a meld operation without copying all the data from either argument. Let $\alpha_{\tau}$ be the type of a persistent data structure supporting a melding operation

$$
\oplus: \alpha_{\tau} \times \alpha_{\tau} \rightarrow \alpha_{\tau}
$$

${ }^{21}$ core.rrb-tree: https://github.com/clojure/core.rrb-vector 
we can define the following three corresponding transient operations when combined with its transient $\alpha_{\tau}^{!}$

$$
\begin{aligned}
& \oplus_{l}^{!}: \alpha_{\tau}^{!} \times \alpha_{\tau} \rightarrow \alpha_{\tau}^{!} \\
& \oplus_{r}^{!}: \alpha_{\tau} \times \alpha_{\tau}^{!} \rightarrow \alpha_{\tau}^{!} \\
& \oplus_{b}^{!}: \alpha_{\tau}^{!} \times \alpha_{\tau}^{!} \rightarrow \alpha_{\tau}^{!}
\end{aligned}
$$

These are produced by taking a transient in either or both arguments. These operations naturally map to an r-value based interface, but it can also be modelled using mutable l-values, as shown in listing 6.3. Note that in the l-value version the last two methods are fundamentally the same, provided for symmetry.

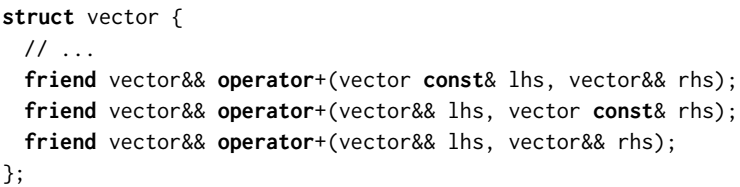

(a) Using r-value transients

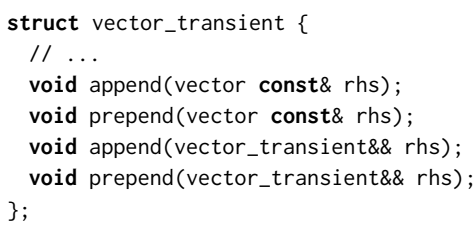

(b) Using mutable transient types

Listing 6.3. Modeling transient concatenation in $\mathrm{C}_{++}$

6.4.3 Transient Concatenation. Implementing transient concatenation does not require one to implement the three meld transient functions separately. The trick is to carry three owner identifiers, that of the left side, that of the right side, and that of the new center nodes. When a node is created or adopted it is assigned the center identifier, which is the identifier of the resulting tree. The center identifier should be that of the left tree for $\oplus_{l}^{!}$and that of the right tree for $\oplus_{r}^{!}$. For $\oplus_{b}^{!}$, we can choose either side based on some heuristic-we propose taking that of the bigger tree. Algorithm 6.2 provides a general skeleton for transient meld operations.

Note that transient concatenation is only barely faster than normal concatenation. The meat of the algorithm happens in the rebalancing step. In the transient version, objects are reused when possible (saving allocations) but the data needs to be moved or copied around anyways. In order to reuse nodes we add complexity (more branching, recursion parameteres, etc.) that expends most cycles that we save by avoiding allocations.

Furthermore, implementing this operation with manual reference counting is particularly tricky because we destroy the old tree as we go, making it hard to keep an account of which nodes to inc() or $\operatorname{dec}()$. Note that with reference counting we can instead keep a free-list of recently deallocated objects (and we do with the default memory policy)-we save allocations yet keep a simpler recursion, often being faster. Also, with reference counting, the transient concatenation algorithm is not required to keep an account of transient ownership. Thus, when using reference counting, our implementation concatenates small trees in-place when only the tail is affected, but resorts to the persistent concatenation algorithm for the general case.

However, we do enable transient concatenation when automatic garbage collection and id-based ownership are used. Otherwise we would not tag the produced and adopted nodes with the identifier of the new owning transient. Failing to do so pessimizes updates later performed on the resulting transient value. 

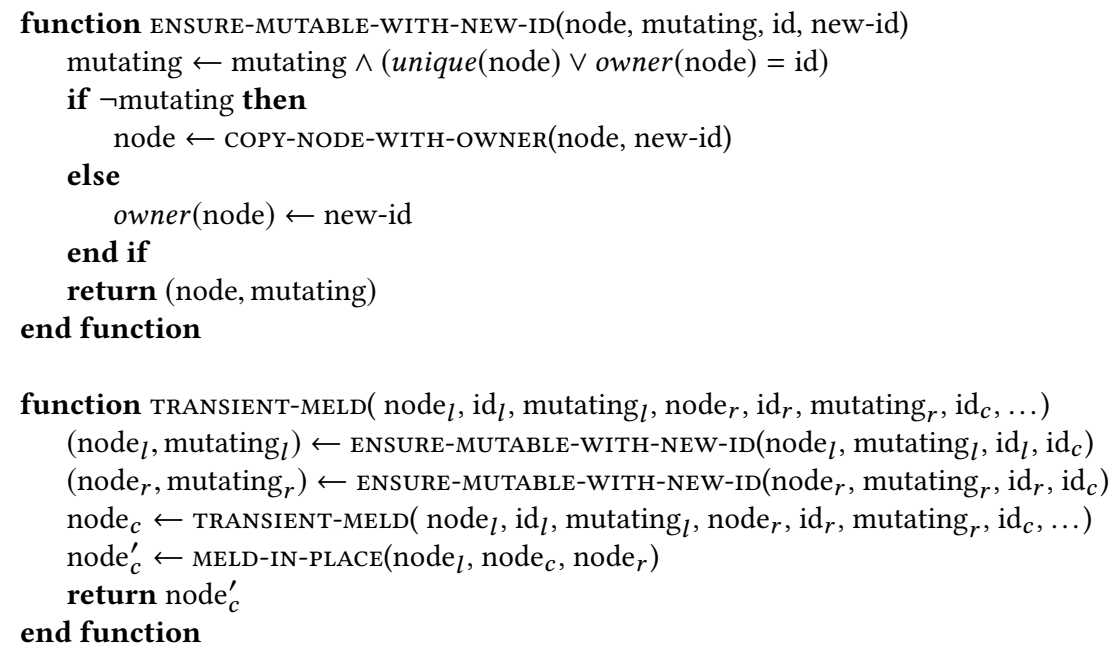

$$
\begin{aligned}
& a_{l} \oplus_{l}^{!} a_{r}=\text { TRANSIENT-MELD }\left(\operatorname{root}\left(a_{l}\right), \mathrm{T}, i d\left(a_{l}\right), \operatorname{root}\left(a_{r}\right), \perp, i d_{\text {noone }}, i d\left(a_{l}\right)\right) \\
& a_{l} \oplus_{r}^{!} a_{r}=\operatorname{TRANSIENT-MELD}\left(\operatorname{root}\left(a_{l}\right), \perp, i d_{n o o n e}, \operatorname{root}\left(a_{r}\right), \mathrm{\top}, i d\left(a_{l}\right), i d\left(a_{l}\right)\right) \\
& a_{l} \oplus_{b}^{!} a_{r}=\operatorname{TRANSIENT-MELD}\left(\operatorname{root}\left(a_{l}\right), \mathrm{T}, i d\left(a_{l}\right), \operatorname{root}\left(a_{r}\right), \mathrm{T}, i d\left(a_{r}\right), i d\left(\operatorname{choose}\left(a_{l}, a_{r}\right)\right)\right)
\end{aligned}
$$

Algorithm 6.2. Structure of a transient meld operation

\section{EVALUATION}

\subsection{Methodology}

We evaluated various implementations (table 1) by running several benchmarks in a specific system (tables 2 and 3). We run each benchmark for three problem sizes $N$ (normally, this is the size of the vector). For practical reasons, we take less samples of bigger problem sizes (table 4).

We run $\mathrm{C} / \mathrm{C}++$ benchmarks using the Nonius framework, ${ }^{22}$ Clojure benchmarks using $\mathrm{Criterium},{ }^{23}$ Python using PyTest. Benchmark, ${ }^{24}$ and Scala using ScalaMeter. ${ }^{25}$ The two first frameworks are based on the Haskell Criterion framework ${ }^{26}$ which introduces interesting statistical bootstrapping methods for the detection of outliers. The rest also do some form of outlier detection. All of them do appropriate measurement of the clock precision and run each benchmark enough times per sample to obtain significant results. The JVM based frameworks take care of minimizing the impact of the garbage collector in the measurements, as well as ensuring that the code is properly JIT-compiled [Georges et al. 2007]. In $\mathrm{C}++$, we manually trigger a full $\mathrm{libgc}$ collection before each benchmark to avoid remaining garbage from previous benchmarks impacting the performance. We also considered disabling the garbage collector during the measurement, but this is impracticable for big problem sizes.

\subsection{Results}

The benchmark results are presented in tables 5 to 8 .

\footnotetext{
$\overline{{ }^{22} \text { https://nonius.io/ }}$

${ }^{23}$ https://github.com/hugoduncan/criterium/

${ }^{24}$ https://github.com/ionelmc/pytest-benchmark

${ }^{25} \mathrm{http}: / /$ scalameter.github.io/

${ }^{26} \mathrm{http}$ ://hackage.haskell.org/package/criterion
} 
Table 1. Evaluated implementations

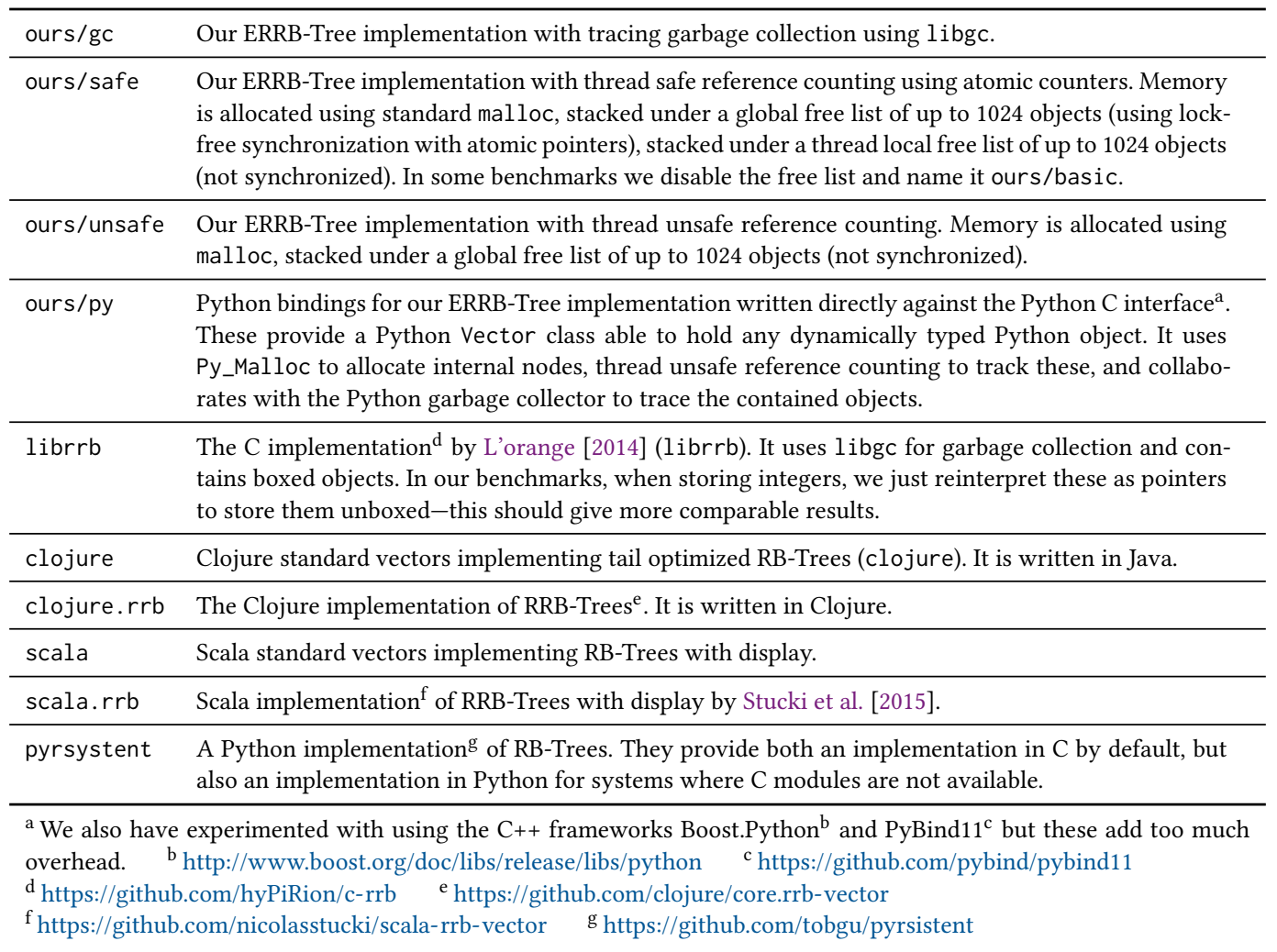

Table 2. System hardware

Table 3. System software

Table 4. Problem sizes

\begin{tabular}{|c|c|c|c|c|c|c|}
\hline Processor & Intel Core i5-460M (64bit) & OS & Linux 4.9.0 (Debian) & Size & $N$ & Samples \\
\hline Frequency & $2.53 \mathrm{GHz}$ & Compiler & gec 6.3 .0 & Small (S) & $10^{3}$ & 100 \\
\hline L1 Cache (per core) & $2 \times 32 \mathrm{~KB}$ (8-way assoc.) & Java & openjdk 1.8.0-121 & Medium (M) & $10^{5}$ & 20 \\
\hline $\begin{array}{l}\text { L2 Cache (per core) } \\
\text { L3 Cache }\end{array}$ & $\begin{array}{l}256 \mathrm{~KB} \text { (8-way assoc.) } \\
3 \mathrm{MB} \text { (12-way assoc.) }\end{array}$ & $\begin{array}{l}\text { Python } \\
\text { Scala }\end{array}$ & $\begin{array}{l}\text { cpython } 2.7 .3 \\
\text { scala } 2.11 .11\end{array}$ & $\begin{array}{l}\text { Mearum (M) } \\
\text { Large (L) }\end{array}$ & $\begin{array}{l}10 \\
10^{7}\end{array}$ & 3 \\
\hline RAM & 4 GB DDR3 $(1,066 \mathrm{MHz})$ & Clojure & clojure 1.8 .0 & & & \\
\hline
\end{tabular}

7.2.1 Abstraction Cost. Our implementation uses various abstraction mechanisms $(\S 4.4, \S 5.2)$. We argued that these are zero-cost abstractions-or may even incur a negative cost when used to remove metadata. We can evaluate this by comparing our implementation with librrb and pyrsistent, since both are written in $\mathrm{C}$ using similar optimizations (off-tree tail). Our Python bindings are faster than pyrsistent in all benchmarks. Our implementation (when combined with libgc) is faster than librrb in most benchmarks excepting two.

librrb supports faster transient random updates (table 7) and shows a speedup of around $20 \%$ because their implementation does not support exceptions (it is plain $\mathrm{C}$ after all) nor recovers gracefully from memory exhaustion. Their update function is thus just a simple loop while, in order to be exception safe, our implementation uses non-tail recursion, executing all potentially failing operations first and only then mutating the tree. 
Table 5. Access Benchmarks. The sum of all values in a $n$ element vector is computed. Elements are accessed either by sequential indexes (e.g. via operator []), iterators, or via internal iteration (i.e. higher order reduce function).

\begin{tabular}{|c|c|c|c|c|}
\hline & & 10ns $\mid \mathbf{S}$ & $\mu \mathrm{s} \mid \mathbf{M}$ & $100 \mu \mathrm{s} \mid \mathbf{L}$ \\
\hline \multirow{8}{*}{$\begin{array}{l}\text { y } \\
\text { z } \\
\text { â⿴囗十 } \\
\text { z }\end{array}$} & ours & 595 & 828 & 851 \\
\hline & relaxed ours & 497 & 930 & 1573 \\
\hline & librrb & 466 & 905 & 1268 \\
\hline & relaxed librrb & 978 & 1280 & 3302 \\
\hline & ours/python & 5412 & 5973 & 7052 \\
\hline & pyrsistent & 5794 & 6189 & 7131 \\
\hline & scala & 9437 & 1546 & 1739 \\
\hline & scala.rrb & 9500 & 1167 & 1360 \\
\hline \multirow{4}{*}{ 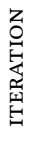 } & ours & 120 & 126 & 144 \\
\hline & relaxed ours & 88 & 113 & 221 \\
\hline & scala & 17368 & 772 & 562 \\
\hline & scala.rrb & 17879 & 629 & 559 \\
\hline \multirow{8}{*}{ 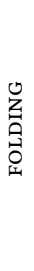 } & ours & 29 & 36 & 75 \\
\hline & relaxed ours & 32 & 38 & 109 \\
\hline & scala & 13904 & 1033 & 943 \\
\hline & scala.rrb & 14835 & 1049 & 941 \\
\hline & clojure & 5122 & 5578 & 6902 \\
\hline & clojure.rrb & 14626 & 19264 & 23533 \\
\hline & std: : vector & 19 & 21 & 56 \\
\hline & std: :list & 210 & 465 & 581 \\
\hline
\end{tabular}

Table 6. ApPEND BENCHMARKs. A $n$ element vector is produced by sequentially appending $n$ elements. In the transient version a mutable interface is used.

\begin{tabular}{|c|c|c|c|c|}
\hline & & $\mu \mathrm{s} \mid \mathbf{S}$ & $100 \mu \mathrm{s} \mid \mathbf{M}$ & $10 \mathrm{~ms} \mid \mathbf{L}$ \\
\hline \multirow{11}{*}{ 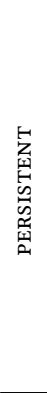 } & ours/basic & 136 & 140 & 172 \\
\hline & ours/safe & 71 & 76 & 98 \\
\hline & ours/unsafe & 31 & 30 & 34 \\
\hline & ours/gc & 48 & 73 & 139 \\
\hline & librrb & 76 & 106 & 184 \\
\hline & ours/python & 214 & 221 & 238 \\
\hline & pyrsistent & 332 & 313 & 338 \\
\hline & scala & 143 & 65 & 159 \\
\hline & scala.rrb & 175 & 64 & 151 \\
\hline & clojure & 107 & 117 & - \\
\hline & clojure.rrb & 246 & 257 & - \\
\hline \multirow{9}{*}{ 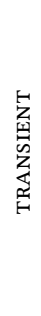 } & ours/basic & 7 & 7 & 8 \\
\hline & ours/safe & 6 & 6 & 8 \\
\hline & ours/unsafe & 5 & 6 & 7 \\
\hline & ours/gc & 5 & 5 & 6 \\
\hline & librrb & 9 & 9 & 12 \\
\hline & clojure & 65 & 66 & - \\
\hline & clojure.rrb & 84 & 85 & - \\
\hline & std: : vector & 4 & 3 & 8 \\
\hline & std: : list & 56 & 56 & 58 \\
\hline
\end{tabular}

Table 7. UpdATE BENCHMARKs. Every element in a $n$ element vector is updated using sequential indexes. In the transient version, a mutable interface is used without destroying the initial value.

\begin{tabular}{|c|c|c|c|}
\hline & $\mu \mathrm{s} \mid \mathbf{S}$ & $100 \mu \mathrm{s} \mid \mathbf{M}$ & $10 \mathrm{~ms} \mid \mathbf{L}$ \\
\hline \multicolumn{4}{|l|}{ PERSISTENT } \\
\hline ours/basic & 459 & 1385 & 2269 \\
\hline ours/safe & 327 & 1200 & 2004 \\
\hline ours/unsafe & 76 & 350 & 667 \\
\hline ours/gc & 251 & 409 & 1226 \\
\hline relaxed ours/basic & 519 & 1549 & 2514 \\
\hline relaxed ours/safe & 380 & 1335 & 2249 \\
\hline relaxed ours/unsafe & 135 & 445 & 891 \\
\hline relaxed ours/gc & 182 & 441 & 828 \\
\hline librrb & 288 & 551 & 1049 \\
\hline relaxed librrb & 316 & 615 & 1238 \\
\hline ours/python & 419 & 732 & 994 \\
\hline pyrsistent & 605 & 884 & 1140 \\
\hline scala & 136 & 64 & 144 \\
\hline scala.rrb & 103 & 68 & 158 \\
\hline clojure & 359 & 626 & - \\
\hline clojure.rrb & 542 & 883 & - \\
\hline \multicolumn{4}{|l|}{ TRANSIENT } \\
\hline ours/basic & 14 & 31 & 41 \\
\hline ours/safe & 14 & 31 & 40 \\
\hline ours/unsafe & 15 & 31 & 40 \\
\hline ours/gc & 17 & 38 & 51 \\
\hline relaxed ours/basic & 15 & 31 & 40 \\
\hline relaxed ours/safe & 14 & 31 & 40 \\
\hline relaxed ours/unsafe & 15 & 31 & 41 \\
\hline relaxed ours/gc & 17 & 38 & 50 \\
\hline librrb & 13 & 21 & 28 \\
\hline relaxed librrb & 19 & 25 & 40 \\
\hline clojure & 114 & 149 & - \\
\hline clojure.rrb & 270 & 340 & - \\
\hline std: : vector & 1 & 3 & 5 \\
\hline
\end{tabular}

Table 8. CONCATE BENCHMARKs. A $n$ element vector is produced by concatenating 10 equally sized vectors.

\begin{tabular}{lrrrr}
\hline & & $\mu \mathrm{s} \mid \mathbf{S}$ & $\mu \mathrm{s} \mid \mathbf{M}$ & $\mu \mathrm{s} \mid \mathbf{L}$ \\
\hline \multirow{2}{*}{ PERSistent } & ours/basic & 7 & 30 & 159 \\
& ours/safe & 5 & 26 & 206 \\
& ours/unsafe & 3 & 16 & 158 \\
& ours/gc & 4 & 16 & 122 \\
& librrb & 8 & 21 & 103 \\
& scala.rrb & 58 & 221 & 857 \\
& clojure.rrb & 1133 & 492 & - \\
\hline \multirow{2}{*}{ TRANSIENT } & ours/gc & 4 & 15 & 112 \\
\hline
\end{tabular}


Table 8 ( $L$ column) shows an example in which librrb does faster concatenation, while our implementation seems faster in all others. Note that we are comparing vectors of normal int values. These are 32 bit in size while pointers are 64 bit wide, thus $B_{l}=6$ because of embedding. The resulting relaxed structures are not the same and in this particular instance we happen to need more rebalancing at the leaves. When repeating the benchmark controlling for $B_{l}$, our implementation provides a consistent $50 \%$ speedup because it uses positions to avoid allocating auxiliary center nodes.

7.2.2 Abstraction Suitability. One of our main goals was to offer sufficiently customizable memory management such that our implementation could be integrated in other language runtimes. In a few hours we had an initial integration with the Python, supporting cooperative garbage collection and allocating memory in the idiomatic ways suggested by the interpreter documentation. These bindings are already faster than pyrsistent, a $\mathrm{C}$ implementation manually tailored against the Python interpreter that implements similar optimizations: it keeps the tail off-tree, uses single threaded reference counting, and it keeps a free list of recently released nodes.

7.2.3 Embedding Effectiveness. While embedding provides some advantage in most operations, its benefits are most evident when accessing the elements. In our implementation we provide three ways of accessing the elements: (1) querying an element by index, (2) using iterators (3) using reduce (i.e. folding). The three methods are compared in table 5.

When using the reduce method, our implementation is only $50 \%$ slower than a fully contiguous std: : vector. This is an excellent result, considering how efficient contiguous arrays are. That method uses internal iteration-this is, it traverses the data-structure once, applying a given operation on the elements. Using templates, the operation can be inlined in the traversal.

External iteration adds some overhead. At every step the iterator needs to check if it is done running through the current leaf, and when it is, it needs to traverse down the tree to find the next leaf. The problem of external iteration over hierarchical data structures is further discussed by Austern [2000]. Still, thanks to embedding, iterating over an ERRB-Vector is a few times faster than iterating over a std: : list.

Comparing the access performance across languages is hard to do in a fair way, because other languages have unrelated costs due to some other features. For example, Clojure's dynamism taxes dealing with basic types ${ }^{27}$. Still, in spite of the best efforts of the JVM JIT, folding C++ EERB-Trees seems orders of magnitude faster than folding in any of the Java based languages, and random access is still about twice as fast.

7.2.4 Transience Effectiveness. Looking at tables 7 and 6 we see that transient updates are an order of magnitude faster than their immutable counterparts.

In fact, for large data sets appends are even faster in a transient ERRB-Vector than in a standard mutable vector (table 6, column $L$ ). We believe that this is due to cache utilization. Even though a standard mutable vector uses exponential growth to support amortized $O(1)$ appends, in the growth step it needs to copy all the data. When the vector does not fit in the cache it needs to load it from main memory. RRB-Vector appends only touches the rightmost path of the tree, which fits in $\mathrm{L} 1$ cache even for huge vectors.

Mutating transient operations are still an order of magnitude slower than on a std: : vector. In this case, the RRB-Tree update cost is dominated by the lookup. Note that we deliberately tested ordered updates to put our implementation against the wall. We saw in informal experiments that when performing the updates in random order, a mutable vector slows down an order of magnitude

\footnotetext{
${ }^{27}$ Clojure supports monomorphic vectors for basic types using (vector-of : type). We tried that method and, surprisingly, found it to actually be slower than generic vectors. Thus we decided not to include it in the presented data
}

Proc. ACM Program. Lang., Vol. 1, No. ICFP, Article 16. Publication date: September 2017. 
for big data sets. Another way to bring RRB transient updates closer to those on mutable purely sequential data would be to provide mutable iterators.

Note that while Scala updates are very efficient compared to immutable updates on other implementations. This is because the display optimization ( $\S 2.3 .4)$ achieves amortized $O(1)$ updates when applied sequentially. However, the display management adds some cost to other operations. Transient updates, although less general, are still significantly faster and can optimize non-local updates.

7.2.5 Considering Reference Counting. While reference counting is the most convenient garbage collection for the $\mathrm{C}++$ developer, they are believed to be inadequate for the implementation of immutable data structures. For arbitrarily deep data structures (e.g. lists), they may overflow the stack when releasing a big object. This is not a problem for RRB-Trees. But an RRB update touches up to $M \times \log _{M}(n)$ reference counts. In a multi-threaded system these updates must be atomic, adding additional overhead. This is also relatively cache inefficient, because the reference counted object needs to be visited in order to update its count. These may also cause shadow writes to the immutable data neighbouring the reference count, limiting the parallelism of concurrent reads.

However, reference counting also opens opportunities. Because it reclaims the memory deterministically, we can put the freed nodes in a free list for future reuse. When performing batches of immutable updates, not only does this avoid calling the relatively slow malloc, but also reuses buffers that have been used recently, most probably paged in and in cache. Our benchmarks show that combining single-threaded reference counting with free lists (even though they are small) provide the best performance for all manipulation operations.

However, multi-threaded reference counting impacts immutable update performance, adding up to a $1.5 X-2 X$ overhead over using 1 ibgc. ${ }^{28}$ For many use cases this might be tolerable, considering the gains across all other operations. But more importantly, reference counting enables transient updates on r-values (§ 6.3). When using move semantics in a disciplined manner across a whole system, how much data is copied during updates depends on the level of aliasing. In other words: this opens a continuum between immutability and mutability, where update performance is characterized by the actual dynamic amount of persistence in the system, even when the programmer uses only immutable interfaces.

We believe that for many realistic workloads this will provide a significant advantage to reference counting over automatic garbage collection. However, we found no way to design an unbiased benchmark to test this hypothesis. Still, our implementation provides a good framework to validate this assumption in concrete real world systems. Users can measure the impact of different memory management configurations in their system and pick the one that fits best.

\section{CONCLUSION}

We described an implementation of ERRB-Trees in $\mathrm{C}++$. By storing unboxed values and supporting transient operations, its performance is comparable to that of mutable arrays while providing optional persistence, and logarithmic concatenation and slicing. Via generic programming and policy-based design, different memory management strategies can be used such that the data structures can be made available to higher level languages through their $\mathrm{C}$ runtimes. Also, when using reference counting and move semantics, all r-values become eligible for transient optimizations. This effectively blurs the boundaries between immutable and mutable values and enables better system wide performance without sacrificing a functional programming style.

\footnotetext{
${ }^{28}$ Informal experiments on a more recent Skylake Intel processor show that the gap actually increases in modern machines, up to a $3 X-4 X$ difference.
} 
We showed that a systems programming language is suitable for implementing immutable data structures. We hope that this helps making these data structures accessible to a wider audience, enabling functional architectures to face the challenge of building highly concurrent and interactive systems.

\section{FUTURE WORK}

Associative containers. We would like to apply the methodology and techniques developed in this work to other persistent data structures. Specially interesting are other wide-node trie based random access containers, like HAMT [Bagwell 2001] and CHAMP [Steindorfer and Vinju 2015] We anticipate that the relatively sparse nature of those data-structures (compared to RRB-Vectors) makes some optimizations more costly $(\S 6.3 .4)$ and alternatives need to be developed. Also, the radix-balanced structure could be used to implement persistent compile-time indexed hybrid structures like those in Boost.Fusion ${ }^{29}$ or Hana ${ }^{30}$

Diffing and patching. Because of structural sharing, comparing persistent data structures is already relatively efficient. It is interesting to compute the differences between two versions to from a patch that can be used to reconstruct the more recent version from an older one. Applications include: serializing a history of transactions to disk or the network, efficiently updating user interfaces, or implementing version control [Demaine et al. 2010].

Applications. We shall explore how RRB-Vectors can be used to design novel architectures, beyond the obvious ones (e.g document as a value). For example, games often use flat data models with entities factored out horizontally into subsystems, with components stored in big per subsystem sequences-data-oriented design [Fabian 2013]. RRB-vectors could be used to design persistent high-performance in-memory data-bases for highly interactive systems.

\footnotetext{
$\overline{{ }^{29} \mathrm{http} / / / \text { boost.org/libs/fusion }}$

${ }^{30}$ http://boost.org/libs/hana
} 


\section{A A POSITION FRAMEWORK}

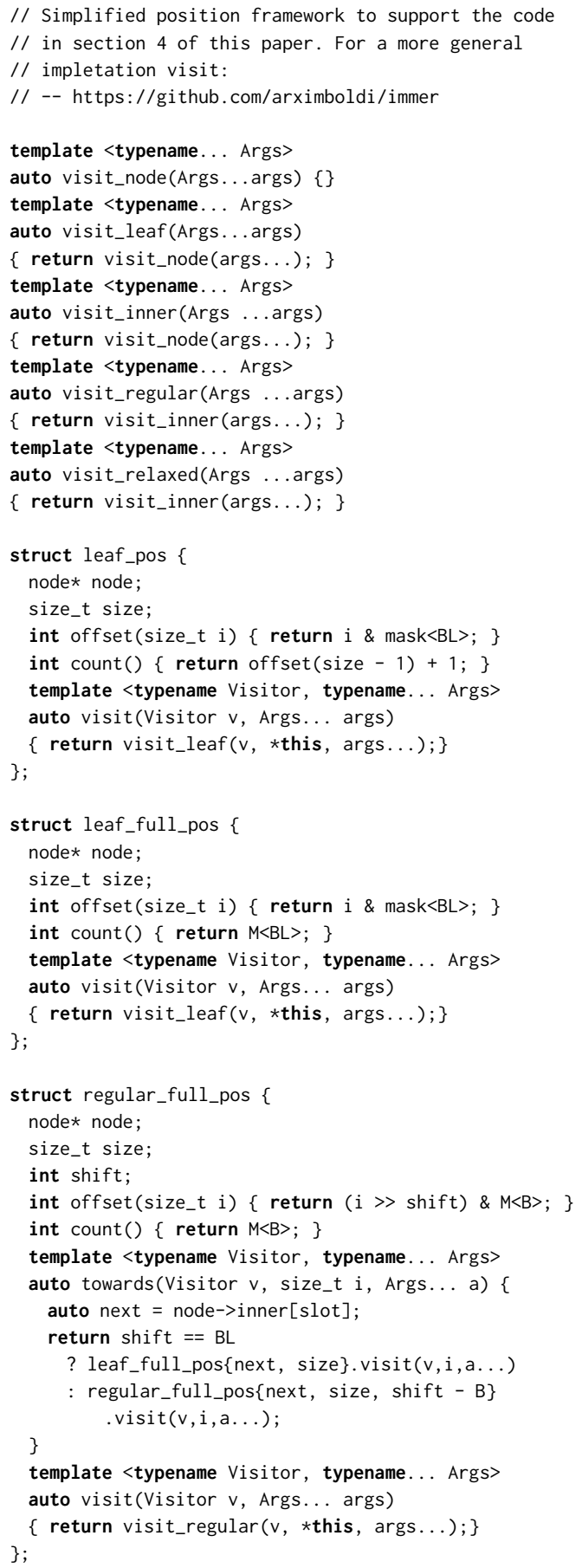

// Simplified position framework to support the code // in section 4 of this paper. For a more general // impletation visit:

// -- https://github.com/arximboldi/immer

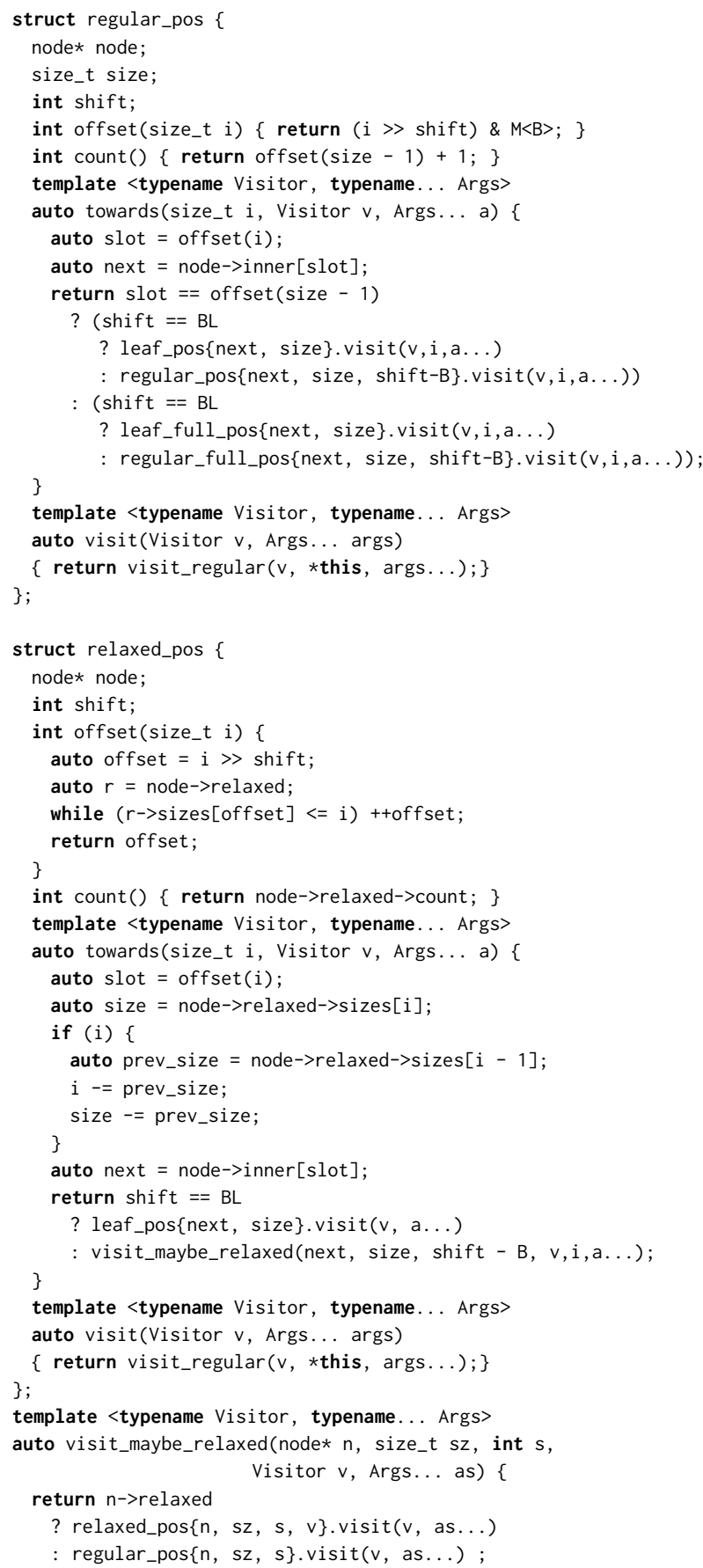


Hans-Juergen Boehm and Mark Weiser. 1988. Garbage Collection in an Uncooperative Environment. Softw., Pract. Exper. 18, 9 (1988), 807-820. https://doi.org/10.1002/spe.4380180902

Hans-J. Boehm, Russ Atkinson, and Michael Plass. 1995. Ropes: An Alternative to Strings. Softw. Pract. Exper. 25, 12 (Dec. 1995), 1315-1330. https://doi.org/10.1002/spe.4380251203

H-J Boehm, M Spertus, and C Nelson. 2008. N2670: Minimal support for garbage collection and reachability-based leak detection (revised. (2008).

Sébastien Collette, John Iacono, and Stefan Langerman. 2012. Confluent Persistence Revisited. In Proceedings of the Twentythird Annual ACM-SIAM Symposium on Discrete Algorithms (SODA '12). Society for Industrial and Applied Mathematics, Philadelphia, PA, USA, 593-601. http://dl.acm.org/citation.cfm?id=2095116.2095166

Erik D. Demaine, Stefan Langerman, and Eric Price. 2010. Confluently Persistent Tries for Efficient Version Control. Algorithmica 57, 3 (July 2010), 462-483. https://doi.org/10.1007/s00453-008-9274-z

Ulrich Drepper. 2008. What Every Programmer Should Know About Memory. Technical Report. Red Hat. http://people. redhat.com/drepper/cpumemory.pdf

J R Driscoll, N Sarnak, D D Sleator, and R E Tarjan. 1986. Making Data Structures Persistent. In Proceedings of the Eighteenth Annual ACM Symposium on Theory of Computing (STOC '86). ACM, New York, NY, USA, 109-121. https://doi.org/10. $1145 / 12130.12142$

Richard Fabian. 2013. Data-Oriented Design. (2013). http://www.dataorienteddesign.com/dodmain/dodmain.html

Amos Fiat and Haim Kaplan. 2001. Making Data Structures Confluently Persistent. In Proceedings of the Twelfth Annual ACM-SIAM Symposium on Discrete Algorithms (SODA '01). Society for Industrial and Applied Mathematics, Philadelphia PA, USA, 537-546. http://dl.acm.org/citation.cfm?id=365411.365528

Matthew Flatt and PLT. 2010. Reference: Racket. Technical Report PLT-TR-2010-1. PLT Design Inc. https://racket-lang.org/ $\operatorname{tr} 1 /$.

Mark Galassi, Jim Blandy, Gary Houston, Tim Pierce, Neil Jerram, Martin Grabmüller, and Andy Wingo. 2002. Guile Reference Manual. (2002). https://www.gnu.org/software/guile/manual/guile.html

Erich Gamma, Richard Helm, Ralph E. Johnson, and John Vlissides. 1995. Design Patterns. Elements of Reusable ObjectOriented Software. Addison-Wesley.

Andy Georges, Dries Buytaert, and Lieven Eeckhout. 2007. Statistically Rigorous Java Performance Evaluation. SIGPLAN Not. 42, 10 (Oct. 2007), 57-76. https://doi.org/10.1145/1297105.1297033

Matthias Grimmer, Chris Seaton, Thomas Würthinger, and Hanspeter Mössenböck. 2015. Dynamically Composing Languages in a Modular Way: Supporting C Extensions for Dynamic Languages. In Proceedings of the 14th International Conference on Modularity (MODULARITY 2015). ACM, New York, NY, USA, 1-13. https://doi.org/10.1145/2724525.2728790

Rich Hickey. 2008. The Clojure Programming Language. In Proceedings of the 2008 Symposium on Dynamic Languages (DLS '08). ACM, New York, NY, USA. https://doi.org/10.1145/1408681.1408682

Howard E. Hinnant, David Abrahams, and Peter Dimov. 2004. A Proposal to Add an Rvalue Reference to the C++ Language Technical Report N1690=04-0130. ISO JTC1/SC22/WG21 - C++ working group.

Ralf Hinze and Ross Paterson. 2006. Finger Trees: A Simple General-purpose Data Structure. Fournal of Functional Programming 16, 2 (2006), 197-217.

Haim Kaplan. 2005. Persistent data structures. In In Handbook On Data Structures And applications, CRC Press 2001, Dinesh Meht And Sarta Sahni (Editors) Boroujerdi, A., And Moret, B.M.E., "Persistency in Computational Geometry"; Proc. 7TH Canadian Conf. Comp. Geometry, Quebeq. 241-246.

Jean Niklas L'orange. 2014. Improving RRB-Tree Performance through Transience. Master's thesis. Norwegian University of Science and Technology.

Nicholas D. Matsakis and Felix S. Klock, II. 2014. The Rust Language. Ada Lett. 34, 3 (Oct. 2014), 103-104. https://doi.org/ 10.1145/2692956.2663188

C. Okasaki. 1999. Purely Functional Data Structures. Cambridge University Press. https://books.google.de/books?id= SxPzSTcTalAC

Aleksandar Prokopec. 2014. Data Structures and Algorithms for Data-Parallel Computing in a Managed Runtime. Ph.D Dissertation. IC, Lausanne. https://doi.org/10.5075/epfl-thesis-6264

Jon Rafkind, Adam Wick, John Regehr, and Matthew Flatt. 2009. Precise Garbage Collection for C. In Proceedings of the 2009 International Symposium on Memory Management (ISMM '09). ACM, New York, NY, USA, 39-48. https://doi.org/ $10.1145 / 1542431.1542438$

Michael J. Steindorfer and Jurgen J. Vinju. 2015. Optimizing Hash-array Mapped Tries for Fast and Lean Immutable JVM Collections. SIGPLAN Not. 50, 10 (Oct. 2015), 783-800. https://doi.org/10.1145/2858965.2814312

Michael J. Steindorfer and Jurgen J. Vinju. 2016. Towards a Software Product Line of Trie-based Collections. In Proceedings of the 2016 ACM SIGPLAN International Conference on Generative Programming: Concepts and Experiences (GPCE 2016). ACM, New York, NY, USA, 168-172. https://doi.org/10.1145/2993236.2993251

Proc. ACM Program. Lang., Vol. 1, No. ICFP, Article 16. Publication date: September 2017. 
Nicolas Stucki, Tiark Rompf, Vlad Ureche, and Phil Bagwell. 2015. RRB Vector: A Practical General Purpose Immutable Sequence. SIGPLAN Not. 50, 9 (Aug. 2015), 342-354. https://doi.org/10.1145/2858949.2784739

D Walker. 2005. Substructural type systems. In In Advanced Topics in Types and Programming Languages. The MIT Press. 